\title{
Mechanodefibrillator dissociation due to prolonged sinus
} \section{arrest}

\author{
Mecano disociación del desfibrilador debido a un paro sinusal prolongado
}

Sasikumar Mahalingam*, Gunaseelan Rajendran, Ajithkumar Rajendran, and Balamurugan Nathan

Department of Emergency Medicine, Jawaharlal Institute of Postgraduate Medical Education and Research, Puducherry, India

To the editor,

Sinus arrest and cardiac arrest are two different terms which are often confused by many. This confusion often leads to inappropriate cardiopulmonary resuscitation (CPR) when patient is connected to defibrillator. Sinus arrest is defined as transient pause in Sino-atrial firing for more than $3 \mathrm{~s}^{1}$. When sinus arrest occurs, other latent pacemakers (atrial myocardium, cells nearby atrioventricular node, and His purkinje system) usually starts firing until Sino-atrial node recover. Sinus arrest can be prolonged till other pacemakers starts firing ${ }^{2}$. Cardiac arrest occurs when these latent pacemakers does not take up the job of alternate firing. Prolonged sinus arrest in a defibrillator may look like a cardiac arrest which might lead to unnecessary CPR.

Here, we would like the put forward a new term "mechano - defibrillator dissociation" which occurs because of prolonged sinus arrest. We should be aware this, so that inappropriate CPR could be avoided. We, emergency physician also faced similar situation while resuscitating a patient because of mechano - defibrillator dissociation caused by prolonged sinus arrest/pseudo cardiac arrest.

A 52-year-old male diabetic, hypertensive, and chronic alcoholic came to our emergency department (ED) with history of giddiness, syncope, and palpitation. On arrival to ED, patient was drowsy, diaphoretic, and hypotensive. Patient was connected to defibrillator which showed a heart rate of $35 / \mathrm{min}$ and saturation was $90 \%$ in room air. ECG showed complete heat block $(\mathrm{CHB})$ and point of care echocardiography showed reduced ejection fraction.

We did a rapid primary assessment and patient was put on oxygen and inotrope support. Patient diagnosis was undifferentiated unstable bradycardia. One group of ED physician was trying to find if any reversible cause of CHB could be there. Another group of ED physician started treating the patient based on bradycardia algorithm. As there was no response with atropine, patient was planned for transcutaneous pacing. Before we could proceed, patient suddenly became fully unconscious. Central pulse was present and POCUS showed cardiac activity. Hence, patient was intubated and transcutaneously paced (60/min heart rate and 30 milliampere) which was used as a bridge to transvenous pacing.

\section{Should we start CPR or not?}

After an hour, to assess patient's intrinsic rhythm, pacing mode was put off which showed a flat line in defibrillator screen.
Correspondence:

*Sasikumar Mahalingam

E-mail: sasiempub@gmail.com DOI: 10.24875/RCCAR.M21000056
Date of acceptance: $17-11-2020$
Disponible en internet: 29-07-2021 Rev Colomb Cardiol. 2021;28(3):297-298 www.rccardiologia.com

CC BY-NC-ND license (http://creativecommons.org/licenses/by-nc-nd/4.0/). 
We did a pulse check and planned for CPR. But before we started it, we noticed a few ventricular escape beats after a prolonged sinus arrest in defibrillator monitor. We did a POCUS which showed cardiac activity which exactly correlated with the ventricular escape beats.

We deferred the plan of CPR and continued with transvenous pacing. Patient was transvenously paced through right internal jugular vein in SSI mode, $80 /$ min heart rate, $5 \mathrm{~mA}$ amplitude and $2 \mathrm{mV}$ sensitivity. Mechanical capture was confirmed with point of care echocardiography. After a few hours of observation, patient regained full consciousness.

Hence, we thought reporting this newer term "mechano - defibrillator dissociation" could help other physician during the resuscitation process.

\section{Funding}

The authors declare have no commercial or financial relationship with any sponsor, or direct professional relationship with it.

\section{Conflicts of interest}

The authors declare that does not exist a conflict of interest.

\section{Ethical disclosures}

Protection of human and animal subjects. The authors declare that no experiments were performed on humans or animals for this study.

Confidentiality of data. The authors declare that they have followed the protocols of their work center on the publication of patient data.

Right to privacy and informed consent. The authors have obtained the written informed consent of the patients or subjects mentioned in the article. The corresponding author is in possession of this document.

\section{References}

1. Viljoen $C$, Chin A, Smith R. Reviewing the causes of electrocardiographic pauses. Cardiovasc J Afr. 2017;28:257-60.

2. Robinson SD, Murray S. Prolonged sinus arrest in an asymptomatic woman. Emerg Med J. 2007;24:613. 